\title{
Sindicalismo magisterial y desempeńo docente en México. Una aproximación empírica
}

\section{Teacher unions and teacher performance in Mexico. An empirical approach}

\section{RESUMEN}

Se diseñó un ejercicio estadístico para medir la asociación entre variables relacionadas con el desempeño docente y la condición de sindicalizado 0 no de los maestros, además de su pertenencia a distintas secciones del SNTE. Los resultados abonan en la idea de una asociación entre el sindicalismo magisterial y ciertos rasgos de los docentes en primarias públicas, tales como los niveles educativos, la formación continua, los controles y evaluaciones del trabajo en las aulas, el uso del tiempo lectivo, y el compromiso docente. Sin embargo, se trata de una asociación mediada, no directa. Se infiere el efecto sindical a través del marco normativo que este actor ayuda a configurar.

Palabras clave: SNTE, CNTE, asociaciones de profesores, política educativa, reforma educativa

Luis Arturo Tapia G.

ABSTRACT

A statistical exercise was designed to measure the association between variables related to teacher performance and unionized or non-union status, as well as belonging to different sections of the SNTE. The results support the idea of an association between teacher unionism and certain features of teachers in public primary schools, such as educational levels, continuous training, classroom work controls and assessments, use of learning time, and the teaching commitment. However, it is a mediated association, not direct. The union effect is inferred through the normative framework that this actor helps to configure.

Key words: SNTE, CNTE, teachers associations, educational policy, educational reform

\section{INTRODUCCIÓN Y PLANTEAMIENTO}

\section{La reforma constitucional en materia educativa en México} (2012-2013) generó una nutrida discusión, ${ }^{1}$ tanto en medios de comunicación como en ámbitos académicos, acerca de la respon-

\footnotetext{
${ }^{1}$ Los cambios en los artículos $3^{\circ}$ y 73 de la Constitución de diciembre del 2012 introdujeron las evaluaciones como mecanismos de acceso, permanencia y ascenso en el servicio docente. De la misma forma, se hizo de esas evaluaciones el mecanismo para la asignación de estímulos salariales. Otro cambio importante fue la redistribución de facultades y roles entre el sindicato de maestros, la autoridad que diseña los criterios de evaluación y las autoridades educativas en los distintos niveles de gobierno (v. gr. la reforma devolvió el manejo de los salarios magisteriales al gobierno federal; excluyó al sindicato de la definición de temas académicos; y amplió las facultades del Instituto Nacional para la Evaluación Educativa, INEE). Finalmente, la reforma facilitó la aplicación de sanciones por el ausentismo de los docentes.

* Catedrático de CONACYT, comisionado en el CIIDIR-IPN, Unidad Oaxaca; latapiagu@ conacyt.mx
} 
sabilidad del Sindicato Nacional de Trabajadores de la Educación (SNTE) en la explicación de los bajos resultados del sistema educativo del país. ${ }^{2}$ De la misma manera, se discutió y se sigue discutiendo acerca de si el tránsito hacia reglas de contratación y promoción de los docentes basadas en la evaluación -como lo plantea la reforma- podría incidir en su desempeño en el aula y con ello en los aprendizajes de los alumnos en escuelas públicas. ${ }^{3}$ En el contexto de estos debates, algunas voces señaladamente críticas del SNTE (v. gr. CCE, 2010) han sostenido que los persistentes bajos resultados educativos del país en pruebas estandarizadas han obedecido a una dinámica de corrupción, opacidad y vetos a las iniciativas de cambio, protagonizada y resguardada por el sindicato de maestros y sus dirigencias tanto nacional como estatales. ${ }^{4}$ Esta dinámica supuestamente impulsada por el SNTE (Rentería y Rubio, 1998) -muchas veces en complicidad con las autoridades educativas locales- habría configurado un sistema tal de reglas de ingreso, permanencia y ascenso en el servicio docente que, con el tiempo, no se generaron mecanismos eficientes de selección ni de formación de maestros; tampoco se establecieron incentivos para su preparación continua ni para su desempeño en el aula, ade-

\footnotetext{
${ }^{2}$ Desde su formación en 1943, el SNTE es el principal sindicato de maestros en México por el número de sus afiliados (más de un millón). Aunque en años recientes han surgido otros sindicatos magisteriales en algunos estados del país resultan de poca relevancia laboral y política.

${ }^{3} \mathrm{~A}$ este respecto se pueden revisar los debates a través de la prensa escrita en los que han estado participando connotados académicos en el campo educativo. Solo por mencionar algunas intervenciones recientes se puede ver Eduardo Backhoff, "La evaluación docente a prueba", en El Universal, 23 de diciembre del 2015 (consulta 15 de enero de 2016); Carlos Ornelas, "Reforma educativa: postura personal", en Excelsior, 3 de febrero del 2016 (consulta 5 de febrero de 2016); Manuel Gil Antón, "Reforma educativa sí, pero no así", en El Universal, 12 de septiembre de 2015 (consulta 15 de enero de 2016). En el mismo sentido, véase el pronunciamiento que un grupo de académicos realizó durante el XIII Congreso Nacional de Investigación Educativa en noviembre del 2015 en la Cd. de Chihuahua, que después se introdujo como una campaña de recolección de firmas en la plataforma Change.org. Entre los firmantes figuran Alberto Arnaut, Ángel Díaz Barriga, María de Ibarrola, Manuel Gil Antón, Imanol Ordorika, Carlos Ornelas y otros (véase: https://www.change.org/p/ sep-mexico-por-una-reforma-educativa-necesaria-y-respetuosa-del-magisterio?source location=petitions_share_skip. Consulta, 15 de enero de 2016).

${ }^{4}$ En este sentido, hacia septiembre del 2012, la asociación civil denominada Mexicanos Primero denunciaba al SNTE y a sus dirigencia como los responsables de la situación del rezago educativo en el país. En un comunicado de prensa la asociación exponía que "El empeño de la cúpula sindical para capturar el sistema de educación pública es una privatización de facto pues controlan el ingreso, la permanencia, la promoción y los estímulos de los maestros, a la par de ejercer su capacidad de veto en el diseño, implementación y evaluación de la política educativa" (ver "Miente cúpula de SNTE: Mexicanos Primero", Sonia del Valle, El Norte, 14 de septiembre, 2012. Consulta 15 de enero de 2016).
} 
más de que no se promovió la rendición de cuentas a la sociedad (CCE, 2010). ${ }^{5}$ En otras palabras, el pretendido manejo clientelar, patrimonialista de las plazas docentes (incluyendo su herencia y venta), la basificación automática de maestros luego de seis meses de ingreso al servicio, ${ }^{6}$ además de los ascensos en la estructura docente bajo reglas escalafonarias que enfatizaban la antigüedad, ${ }^{7}$ generaron una situación que los críticos consideran adversa al desempeño y al mérito docente, además de favorable para el magisterio y desfavorable para alumnos y padres de familia (Andere, 2007; Del Valle, 2008; Martínez y Nieto, 2013; Redacción El Universal, 2013; Villarino, 2014).

En torno a esta postura crítica y antisindical creció en los últimos años una coalición política, social y mediática, cuya agenda educativa, basada en el rediseño de los premios y sanciones en el sistema educativo, fue trasladada a la reforma de diciembre de 2012 y cuyo componente central es la evaluación docente y el condicionamiento de la permanencia y el ascenso en el servicio a los resultados obtenidos en las pruebas. ${ }^{8}$

Más allá de la intensa polarización social y académica que la reforma ha provocado, y de los fuertes afectos y desafectos que le

\footnotetext{
${ }^{5}$ La citada asociación, Mexicanos Primero, acusaba en febrero del 2013 al SNTE de mostrar su talante más conservador al resistir al desmantelamiento de una fuente ilegal de financiamiento que eximía a las dirigencias de la rendición de cuentas, e intentando que todo permaneciera opaco y sin sanciones efectivas. A través de un comunicado de prensa la asociación cuestionaba que, con esta resistencia, el SNTE contradecía la posibilidad de que el sistema público de educación cumpliera su función de combatir la pobreza, la marginación y la ignorancia (ver José Juan Reyes, El Economista. Online Edition, 13 de febrero de 2013).

${ }^{6}$ Histórica y formalmente, el ingreso al servicio docente se diseñó a través de reglas escritas y no escritas en el sistema educativo. Por ejemplo, se hizo común que los egresados de las escuelas de formación de maestros (i. e. las denominadas escuelas Normales) para educación básica ingresaran de forma automática al servicio una vez concluidos los estudios. A su vez, la Ley Federal de Trabajadores al Servicio del Estado (LFTSE) establecía en su artículo 6 que después de seis meses de trabajo se obtenía el contrato de base. De esta manera, los egresados de las escuelas Normales obtenían contratos permanentes en poco tiempo. Aunque cabe agregar que en los últimos años se empezó a generar la figura de los maestros con contratos temporales, en algunos casos cubriendo interinatos.

${ }^{7}$ El Reglamento de Escalafón de los Trabajadores al Servicio de la Secretaría de Educación Pública (SEP), en su Título primero, artículo $1^{\circ}$ define al "escalafón" como el sistema organizado en la SEP para efectuar las promociones de ascenso de los trabajadores de base y autorizar las permutas. Es este sistema el que regula el ascenso de un profesor a puesto de director de escuela y/o al de supervisor de zona.

${ }^{8}$ Esta coalición tendría su postura más paradigmática en los diagnósticos difundidos por Mexicanos Primero (Mexicanos y Fundación, 2007) y por la Organización para la Cooperación y el Desarrollo Económico (OCDE, 2005).
} 
han acompañado, es necesario generar evidencias acerca de la relación entre el sindicalismo magisterial y el desempeño docente.

El supuesto efecto sindical sobre el trabajo de los maestros y su eliminación a través de nuevas regulaciones, parece haber estado en la mira del fuerte componente administrativo-laboral de la reforma de 2012-2013, pero hasta donde se sabe, esto no se sostuvo en evidencias empíricas -ni abundantes ni conocidas en el ámbito académico-que mostraran la asociación entre sindicalismo y desempeño pues, de hecho, se trata de una relación poco estudiada en México. El trabajo que aquí se presenta atiende este vacío analítico abordando tres interrogantes: primero, ¿existen diferencias en el desempeño de maestros sindicalizados y no sindicalizados? Así también, ¿existen diferencias en el desempeño de maestros afiliados a distintas secciones del SNTE, específicamente aquellos que lo están a secciones institucionales (SS.II.) y los afiliados a secciones disidentes como las agrupadas en la CNTE?? Finalmente, ¿existe algo que pudiera denominarse un efecto sindical sobre el desempeño de los docentes?

El trabajo reporta los resultados de un ejercicio estadístico exploratorio con el que se ha intentado identificar y medir la asociación entre el sindicalismo magisterial y el desempeño docente en escuelas primarias de trece entidades del país; ${ }^{10}$ además de

\footnotetext{
${ }^{9}$ El SNTE es un sindicato nacional y se organiza por secciones con presencia estatal. En cada estado existen una, dos o tres secciones del SNTE. La distinción entre las SS.II. y las secciones disidentes se generó desde los años ochenta, en el contexto de la llamada insurgencia magisterial, cuando un gran número de maestros de distintas secciones y de distintos estados del país se movilizaron bajo las demandas de democracia sindical y más salario. Hacia finales de la década perduraron la organización y las movilizaciones magisteriales en algunos estados del sur y del sureste (principalmente Michoacán, Guerrero, Morelos, Chiapas y Oaxaca). Desde esos años las secciones disidentes se han caracterizado por mantener su demanda de democracia sindical, y por plantear agendas de negociación con las autoridades educativas de forma paralela a las impulsadas por la dirigencia nacional. Esas agendas paralelas son respaldadas, generalmente, con intensas movilizaciones en las calles. Estas secciones se agruparon en la denominada Coordinadora Nacional de Trabajadores de la Educación (CNTE) pretendiendo ser un gran frente democratizador del magisterio y de la vida nacional a través de su alianza con otras organizaciones sociales de izquierda (Del Campo, 1992). En contraste, las secciones institucionales se caracterizan por la débil autonomía frente a la dirigencia nacional, lo que se traduce en un casi nulo registro de movilizaciones-protestas y una débil presencia de grupos disidentes en su interior. Cabe observar que la CNTE no tiene una existencia legal o formal. Se trata de una organización de maestros que se autodenomina Coordinadora, pero cuyos integrantes -maestros disidentes- son afiliados formales del SNTE (Cook, 1996; Del Campo, 1992; Luna A. y Arellanes M., 2009).

${ }^{10}$ En México existen 32 entidades federativas. El ejercicio reportado involucra a poco menos de la mitad de estas. Los maestros afiliados al SNTE laboran exclusivamente en el sistema de escuelas públicas en el nivel básico (alumnos de 3 a 15 años de edad) y en el de
} 
buscar la existencia de contrastes entre los modelos sindicales de las SS.II. y de las secciones disidentes. De esta manera, a través de diecinueve variables que la literatura identifica como algunos de los componentes o rasgos del desempeńo docente (Blanco B., 2007; Valenti, Blanco B. y De los Heros, 2007; Valenti, Salazar E., Florez, y Luna, 2009), se han comparado maestros sindicalizados y no sindicalizados y, dentro de los primeros, a maestros pertenecientes a las SS.II. y a los de la sección 22 (S-22) del SNTE, expresión más acabada del sindicalismo disidente. ${ }^{11}$

La evidencia reportada ofrece indicios de diferentes niveles de desempeño entre maestros sindicalizados y no sindicalizados, y dentro de los primeros también se registran diferencias entre maestros de las SS.II. y de la S-22. Sin ser concluyente, estos resultados abonan en la discusión sobre la existencia de un efecto sindical en el desempeño de los docentes.

El documento se estructura en cinco apartados. En el primero se ofrece una exposición del marco teórico, en el segundo se describe la metodología que se siguió para el ejercicio, en el tercero se reportan los resultados obtenidos. En el cuarto apartado se discuten estos resultados. En un apartado final se ofrecen las conclusiones.

\section{MARCO TEÓRICO}

A pesar de la relevancia que cobró el tema en el marco de la reforma educativa, y de la presencia de un actor sindical casi predominante en el sistema educativo, no se tienen identificados en México estudios empíricos sobre la asociación entre sindicalismo magisterial y desempeño docente. ${ }^{12} \mathrm{El}$ antecedente de discusión

media superior (alumnos de 15 a 18 años). El primer sistema se desagrega en tres subniveles: educación preescolar, primaria y secundaria. El estudio se centró en la educación primaria, frecuentada por alumnos de entre 6 y 12 años de edad.

${ }^{11} \mathrm{La} \mathrm{S}-22$ se ha caracterizado por representar el proyecto de disidencia magisterial mejor logrado. A diferencia de otras secciones del SNTE, en la S-22 de Oaxaca durante las últimas dos décadas se fueron desterrando los últimos vestigios del sindicalismo institucional, el cual pervivió dentro de otras secciones disidentes. Algunos trabajos que documentan el caso de la S-22 son Vicente C., 2006; Yescas M., 2008; Yescas M. y Zafra, 2006.

${ }^{12}$ De hecho, este horizonte específico de análisis parece descuidado en el contexto latinoamericano, donde se han desarrollado sobre todo estudios acerca del nexo entre sindicatos docentes y reformas educativas (Braslavsky, 2006; Gallardo, 2006; Gallardo, 2004; Gallardo, 2000; Palamidessi, 2003; Tiramonti, 2001) descuidando el tema planteado en este trabajo. Por mencionar algunos. 
más cercano se registra en el ámbito académico estadounidense, donde el tema ha generado numerosos trabajos desde los ochenta (Coulson, 2010; Dan C., 2008; Eberts, Hollenbeck y Stone, 2002; Eberts y Stone, 1987; Grimes y Register, 1990; Hannaway y Rotherham, 2008; Hess y West, 2006; Howard, Rosen y Powell, 1996; Hoxby, 1996; Kurth, 1987; Milkman, 1997; Tucker, 2011). ${ }^{13}$

Esta literatura ofrece evidencia y elementos analíticos que ayudan a pensar el caso mexicano. ${ }^{14}$ En la misma se indica que existen factores dentro del proceso educativo en los que se observa una incidencia relevante del sindicalismo magisterial, pero los argumentos en torno al signo y a la fuerza de su vínculo con el desempeño docente y el logro educativo están divididos (Coulson, 2010; Carini, 2002; Hess, 2006; Moo, 1999). En la literatura es posible identificar argumentos que revisan o cuestionan efectos de los sindicatos docentes que podrían considerarse negativos (Eberts y Stone, 1987; Fuller, Mitchell y Hartmann, 2000; Hannaway y Rotherham, 2008; Ingersoll, Hoxby y Scrupski, 2004; Johnson, 1984; Moo, 1999), mismos que se podrían resumir en los siguientes puntos:

- Los sindicatos de docentes elevan los costos de la educación, con lo que se restringen los recursos destinados a mejorar el logro escolar. En otras palabras, sesgan la distribución de recursos en favor del magisterio.

- Los sindicatos eliminan los incentivos que tienen los maestros

\footnotetext{
${ }^{13}$ La existencia de dos grandes centrales sindicales en las que se agrupan los numerosos sindicatos docentes de E.U., aunado al estancamiento del logro educativo que ha sufrido este país en los últimos años -según revelan las pruebas estandarizadas internacionales-, han generado en los ambientes académicos estadounidenses una gran discusión sobre el tema.

${ }^{14} \mathrm{No}$ se ignora el hecho de que, por muchas razones, el sindicalismo norteamericano no es equiparable al mexicano; especialmente cuando se trata de un sindicato tan singular como lo es el SNTE y un modelo laboral y un régimen sindical tan contrastante con el estadounidense como el mexicano. Baste recordar, por ejemplo, que a diferencia de sus contrapartes estadounidenses, los sindicatos de maestros en México no tienen derecho a la negociación colectiva. Las relaciones de trabajo de los maestros de escuelas públicas en México se rigen por la Ley Federal de los Trabajadores al Servicio del Estado (LFTSE), reglamentaria del apartado B del Artículo 123 Constitucional. Este marco jurídico prohíbe la negociación colectiva para el magisterio. No obstante, en los hechos se ha generado una negociación en la que el sindicalismo funge como interlocutor de la SEP y de las autoridades educativas en los estados. Sin embargo, aquí interesa el caso estadounidense como un antecedente importante en la investigación acerca del sindicalismo magisterial y la educación.
} 
para mejorar la instrucción, por ejemplo: blindando a los profesores ineficaces contra el despido y vinculando los salarios a la antigüedad en lugar de hacerlo al mérito.

- La creciente formalización de los procedimientos como resultado de la sindicalización estorba la habilidad de los directores para manejar sus escuelas. En otras palabras, afecta la gestión escolar y reduce la capacidad de los directivos para tomar decisiones clave y gestionar su cuerpo docente.

- Los sindicatos docentes refuerzan las relaciones de desconfianza entre maestros y directivos; es decir, impactan las relaciones laborales dentro de las escuelas.

- Debido a su influencia política, los sindicatos de maestros pueden bloquear reformas educativas que amenazan sus intereses; es decir, ejercen poderes de veto.

Frente a esta perspectiva crítica (y casi antisindical) la literatura reporta también argumentos orientados a resaltar el sindicalismo magisterial como un hecho positivo en el ámbito educativo (Eberts y Stone, 1987; Fuller et al., 2000; Hannaway y Rotherham, 2008; Ingersoll et al., 2004; Johnson, 1984). Algunos argumentos comunes en esta vertiente sostienen que:

- Los salarios más altos de los maestros sindicalizados y los beneficios asociados con ello atraen y retienen a mejores docentes.

- Los sindicatos ofrecen a los profesores un mayor sentido del profesionalismo y de la dignidad.

- Los sindicatos proporcionan a los maestros una voz colectiva para expresar sus ideas y preocupaciones.

- Los sindicatos mejoran la moral de los profesores y la satisfacción en el trabajo.

- Los sindicatos incentivan prácticas para aumentar el rendimiento estudiantil, por ejemplo, clases más pequeñas y la planificación del tiempo designado para la instrucción.

- Los sindicatos presionan a la gestión y a las escuelas, o a ambos, para convertirse en organizaciones más eficaces.

Según esta segunda vertiente, los sindicatos ayudan en la mejora del desempeño docente por lo que los intereses de los 
maestros y las necesidades educativas de los niños no serían vistas como incompatibles, sino entrelazadas (Eberts et al., 2002; Zigarelli, 1994).

Aunque la literatura y las evidencias están claramente divididas, un hecho relevante que se puede observar es la importancia que ha tomado en los estudios el papel de los sindicatos en el desempeńo docente y en los resultados educativos. También es necesario observar que, a pesar de la distancia entre los modelos sindicales estadounidense y mexicano, emerge una agenda de temas comunes, referidos no tanto a los modelos de regulación laboral o a los sistemas educativos -claramente distintos- sino a las lógicas del quehacer sindical y sus efectos.

La literatura identifica a los sindicatos como actores relevantes dentro de los sistemas educativos y por ello les da un lugar dentro de la agenda de investigación que indaga los resultados educativos, superando hasta cierto punto la distancia que por décadas se mantuvo entre los especialistas interesados en ambas partes de la relación (i. e. sindicalismo y educación).

La citada discusión hace eco de un debate central en los estudios del trabajo durante los ochenta y los noventa a propósito del papel de los sindicatos en la productividad de las empresas (solo por mencionar algunos, Addison y Hirsch, 1989; Allen, 1984; Betcherman, 1991; Bhattacherjee, 1987; Boal y Pencavel, 1994; Bronars, Deere y Tracy, 1994; Brunello, 1992; Clark, 1984; Denny, 1997). Se trata de una discusión a la fecha inconclusa y cuyo estado indica que dicho papel y sus efectos varían según contextos y organizaciones, por lo que no se puede apuntar un efecto negativo o positivo per se de lo sindical en la productividad (Aidt y Tzannatos, 2002). Son necesarios los estudios de caso.

Tal como el papel de los sindicatos en la productividad de las empresas no tiene un status conclusivo dentro de la literatura, tampoco lo tiene la relación entre sindicalismo y desempeño docente. De esta manera, el trabajo se inscribe en esta doble vertiente de estudios.

Más allá de las críticas que se puedan verter hacia el sindicalismo magisterial, es necesario construir evidencias que esclarezcan la naturaleza y efectos de esta organización en el desempeño de los maestros. 


\section{LA METODOLOGÍA}

El ejercicio se diseñó con el uso de diecinueve variables (cuadro 1) de una base de datos de 79 mil casos que contiene las respuestas de los cuestionarios de contexto de la Prueba ENLACE, 2008 aplicados mediante muestreo aleatorio a alumnos de sexto año de primarias públicas generales y de primarias privadas de 13 estados del país así como a profesores y a directores. ${ }^{15}$ Se trata de una base de datos tipo panel en la que se anidan los datos de alumnos, docentes y escuelas.

\section{CUADRO 1. Variables empleadas en el modelo estadístico}

\begin{tabular}{|c|c|c|}
\hline Variables & Escala & Informante \\
\hline \multicolumn{3}{|l|}{ Frecuencia con la que el maestro: } \\
\hline 1) Dedica menos de la mitad del tiempo de clase a la enseñanza & Intervalo & Alumnos \\
\hline 2) Durante las horas de clases se sale del salón & Intervalo & Alumnos \\
\hline 3) Nivel máximo de estudios del docente & Intervalo & Docente \\
\hline 4) Número de cursos de actualización y/o capacitación a los que asistió el año anterior & Intervalo & Docente \\
\hline 5) Ayuda cuando el alumno no entiende algún tema de las clases & Intervalo & Alumnos \\
\hline 6) Permite expresar libremente las opiniones del alumno & Intervalo & Alumnos \\
\hline 7) Toma en cuenta las opiniones de los alumnos para mejorar la enseñanza & Intervalo & Alumnos \\
\hline 8) Motiva al alumno para aprender más y seguir estudiando & Intervalo & Alumnos \\
\hline 9) Mantiene la disciplina del grupo durante las clases & Intervalo & Alumnos \\
\hline 10) Corrige los errores que encuentra en las tareas & Intervalo & Alumnos \\
\hline 11) Escucha y da recomendaciones cuando los alumnos tienen problemas & Intervalo & Alumnos \\
\hline \multicolumn{3}{|c|}{ Frecuencia con la que se evalúa al maestro en los siguientes aspectos: } \\
\hline 12) Dominio de contenido del grado/asignatura & Intervalo & Directores \\
\hline 13) Estrategias de enseñanza-aprendizaje & Intervalo & Directores \\
\hline 14) Capacitación y actualización & Intervalo & Directores \\
\hline 15) Planeación y preparación de clases & Intervalo & Directores \\
\hline 16) Métodos de evaluación del aprendizaje de los estudiantes & Intervalo & Directores \\
\hline 17) Resultado del logro de los estudiantes & Intervalo & Directores \\
\hline 18) Control disciplinario del grupo & Intervalo & Directores \\
\hline 19) Participación en actividades académicas. & Intervalo & Directores \\
\hline
\end{tabular}

\footnotetext{
${ }^{15}$ Los cuestionarios de contexto se aplicaron a la par de la ahora extinta prueba ENLACE. Se trataba de muestras recogidas entre alumnos, padres de familia, docentes y directores, con las que se recababa información acerca de los factores que conforman los contextos educativos. La muestra para la aplicación de los cuestionarios era aleatoria, estratificada y proporcional por modalidad de escuela, por estado y por nivel de desempeño global de la escuela (SEP, 2008).
} 
Se decidió el uso de los datos de la prueba ENLACE, 2008 porque fue el único año en el que la $S-22$ permitió la aplicación de la prueba en casi la totalidad de escuelas de Oaxaca. ${ }^{16}$ No existen otros datos disponibles en los que se pueda incluir el caso de Oaxaca. ${ }^{17}$

Las diecinueve variables utilizadas guardan asociación en la literatura con el desempeño docente (Blanco B., 2007; Fernández A., 2003; Valenti et al., 2007; Valenti et al., 2009). Quince de estas se utilizaron para construir dos índices: un índice de compromiso docente (según percepción de alumnos), ${ }^{18} \mathrm{y}$ un índice escolar de evaluación docente (según percepción de directores). ${ }^{19}$ De esta manera, la base de datos final contiene seis variables (cuadro 2).

\footnotetext{
${ }^{16}$ Se trata de una base de datos depurada, ya que se eliminaron los casos que la misma SEP reporta con probabilidad de copia. La prueba ENLACE ha suscitado controversias a grado tal que se suspendió su aplicación en el 2014 y se volvió a diseñar para su aplicación en el 2015. El INEE firmó un convenio con la Universidad Autónoma de Aguascalientes (UAA) para que un grupo de 10 expertos de México y de otros países llevaran a cabo una revisión de la prueba. Los resultados indicaron que esta presentaba diversas fallas en su validez técnica, cultural, de aplicación y de uso (INEE, 2014). El empleo de los resultados de esta prueba en nuestro ejercicio se justifica con cinco argumentos: en primer lugar, se tuvo el cuidado de excluir casos que la misma SEP marcaba con probabilidad de copia. Segundo, solo se tomaron primarias generales, excluyendo primarias indígenas y del Consejo Nacional de Fomento Educativo (CONAFE), por lo cual el argumento de la falta de pertinencia cultural de la prueba no afecta en los datos. Tercero, en el ejercicio no se trabajó con los resultados de las pruebas estandarizadas, sino solo con los cuestionarios de contexto. Cuarto, la prueba es censal, y en el 2008 se logró aplicar casi en la totalidad de las escuelas oaxaqueñas. Quinto, la base de datos de esta prueba permite desglosar los datos a nivel de aula, algo que en otras bases (Excale, PISA) no es posible. Finalmente, cabe observar que las escuelas de la sección 59 de Oaxaca no fueron evaluadas por la falta de clave de escuela que padecen sus módulos.

${ }^{17}$ Otras pruebas aplicadas en el estado (v. gr. las Excale) no incluyen identificadores que permitan construir una base tipo panel como la aquí referida.

${ }^{18}$ En la generación de este índice se utilizan siete de las variables enunciadas (vid Nota al pie 13): a) la frecuencia con la que el maestro ayuda al alumno cuando este no entiende algún tema; b) la frecuencia con la que el maestro permite expresar libremente opiniones; c) la frecuencia con la que el maestro toma en cuenta las opiniones de los alumnos para mejorar la clase; d) la frecuencia con la que el maestro motiva a aprender y a seguir estudiando; e) la frecuencia con la que el maestro mantiene la disciplina del grupo durante clases; f) la frecuencia con la que el maestro corrige los errores que encuentra en las tareas; y g) la frecuencia con la que el maestro escucha y da recomendaciones cuando el alumno tiene problemas (ver cuadro 1).

${ }^{19}$ Para generar este índice se toman en cuenta ocho variables: la frecuencia con la que se evalúan los siguientes aspectos: dominio de contenido del grado/asignatura, estrategias de enseñanza-aprendizaje, la actualización del docente, la planificación y preparación de clases, los métodos de evaluación del aprendizaje de los estudiantes, el logro de los estudiantes, el control disciplinario del grupo, y finalmente, la participación del docente en actividades escolares (ver detalles del índice en Anexo 1).
} 
SINDICALISMO MAGISTERIAL Y DESEMPEÑO DOCENTE EN MÉXICO...

CUADRO 2. Lista de variables del modelo

\begin{tabular}{|l|l|c|c|c|c|c|}
\hline Nombre de variable & Definición de variable & Tipo de variable & Media & $\begin{array}{c}\text { Desviación } \\
\text { estándar }\end{array}$ & $\begin{array}{c}\text { Valor } \\
\text { mínimo }\end{array}$ & $\begin{array}{c}\text { Valor } \\
\text { máximo }\end{array}$ \\
\hline Mitadtpo & $\begin{array}{l}\text { El maestro dedica menos } \\
\text { de la mitad del tiempo } \\
\text { al aprendizaje }\end{array}$ & Intervalo & 2.33 & 1.68 & 1 & 5 \\
\hline Saleaula & $\begin{array}{l}\text { El maestro sale del salón } \\
\text { en horas de clase }\end{array}$ & Intervalo & 2.46 & 0.46 & 1 & 5 \\
\hline nivesDnt & $\begin{array}{l}\text { Nivel máximo de } \\
\text { estudios del docente }\end{array}$ & Intervalo & 3.42 & 0.97 & 1 & 6 \\
\hline Cursdnte & $\begin{array}{l}\text { Número de cursos } \\
\text { tomados por el docente } \\
\text { en el ciclo }\end{array}$ & Intervalo & 3.19 & 1.17 & 1 & 5 \\
\hline Icompdnte & $\begin{array}{l}\text { Índice de compromiso } \\
\text { docente (percepción de } \\
\text { alumnos) }\end{array}$ & Razón & 4.21 & 0.74 & 0 & 5 \\
\hline levaldnte & $\begin{array}{l}\text { Índice escolar de } \\
\text { evaluación docente }\end{array}$ & Razón & 4.11 & 0.41 & 0 & 5 \\
\hline
\end{tabular}

Fuente: Elaboración propia

Para la generación de los referidos índices se aplicó un análisis factorial confirmatorio (CFA, por sus siglas en inglés) con ayuda del paquete estadístico Mplus, versión 6. Los detalles estadísticos de la generación de los índices se ofrecen en los Anexos 1 y 2.

Utilizando la técnica estadística denominada Análisis de Varianza (ANOVA, por sus siglas en inglés), se compararon las medias de variables relativas al desempeño docente entre maestros sindicalizados y no sindicalizados (i. e. escuelas públicas y privadas), y entre maestros de secciones institucionales y de una sección disidente, todas del SNTE. Para comparar las medias entre pares de categorías se empleó la prueba de Scheffe. En ambos casos se utilizó el paquete estadístico Stata 13.

Partiendo de las preguntas de investigación planteadas (vid Supra), se buscó evidencia estadística para rechazar la hipótesis de que las características de los docentes asociadas al desempeño no registran diferencias significativas en sus medias entre las categorías de maestros estudiados. De esta manera, se plantearon hipótesis nulas de dos colas para cada una de las variables. Estas tienen la forma siguiente: 


$$
H_{0}: \mu_{Y(\text { Privadas })}=\mu_{Y(S-22)}=\mu_{Y(S S . I I)}=\mu_{Y(\text { total })}
$$

Por consiguiente, los efectos principales $=0$; es decir, no existiría relación entre el desempeño docente y la condición de sindicalizado, en primer lugar, y de pertenecer a alguna sección sindical específica.

El ejercicio se realizó con los datos de trece entidades del país (cuadro 3), doce de estas consideradas institucionales y una considerada disidente (i.e. Oaxaca).

CUADRO 3. Listado de entidades incluidas en la base

\begin{tabular}{|l|c|c|c|}
\hline \multicolumn{1}{|c|}{ Entidad } & Alumnos & \% de casos & \% Acumulado \\
\hline Aguascalientes & 6309 & 7.97 & 7.97 \\
\hline Coahuila & 7131 & 9.01 & 16.98 \\
\hline Colima & 3621 & 4.57 & 21.55 \\
\hline Durango & 3364 & 4.25 & 25.8 \\
\hline Guanajuato & 14590 & 18.43 & 44.23 \\
\hline Nayarit & 2343 & 2.96 & 47.19 \\
\hline Nuevo León & 7950 & 10.04 & 57.23 \\
\hline Oaxaca & 4896 & 6.18 & 63.41 \\
\hline Querétaro & 4291 & 5.42 & 68.83 \\
\hline San Luis Potosí & 6011 & 7.59 & 76.43 \\
\hline Sinaloa & 6810 & 8.6 & 85.03 \\
\hline Sonora & 5242 & 6.62 & 91.65 \\
\hline Tamaulipas & 6610 & 8.35 & 100 \\
\hline Total de casos & 79168 & 100 & \\
\hline
\end{tabular}

Fuente: Elaboración propia

El estatus de institucional o de disidente de una sección responde a un doble criterio: por un lado, lo significativa que resulta la presencia de grupos antagónicos a las directrices y estrategias marcadas por el Comité Ejecutivo Nacional (CEN) del SNTE al interior de las secciones y, por otro, la frecuencia con la que en las secciones se recurre a movilizaciones y a repertorios de protesta social como estrategia de negociación con las autoridades educativas. De acuerdo con estos criterios, se identificaron doce entidades en las que se registra una presencia casi nula de disidencia magisterial, además de un escaso uso de movilizaciones (gráfica 1). 


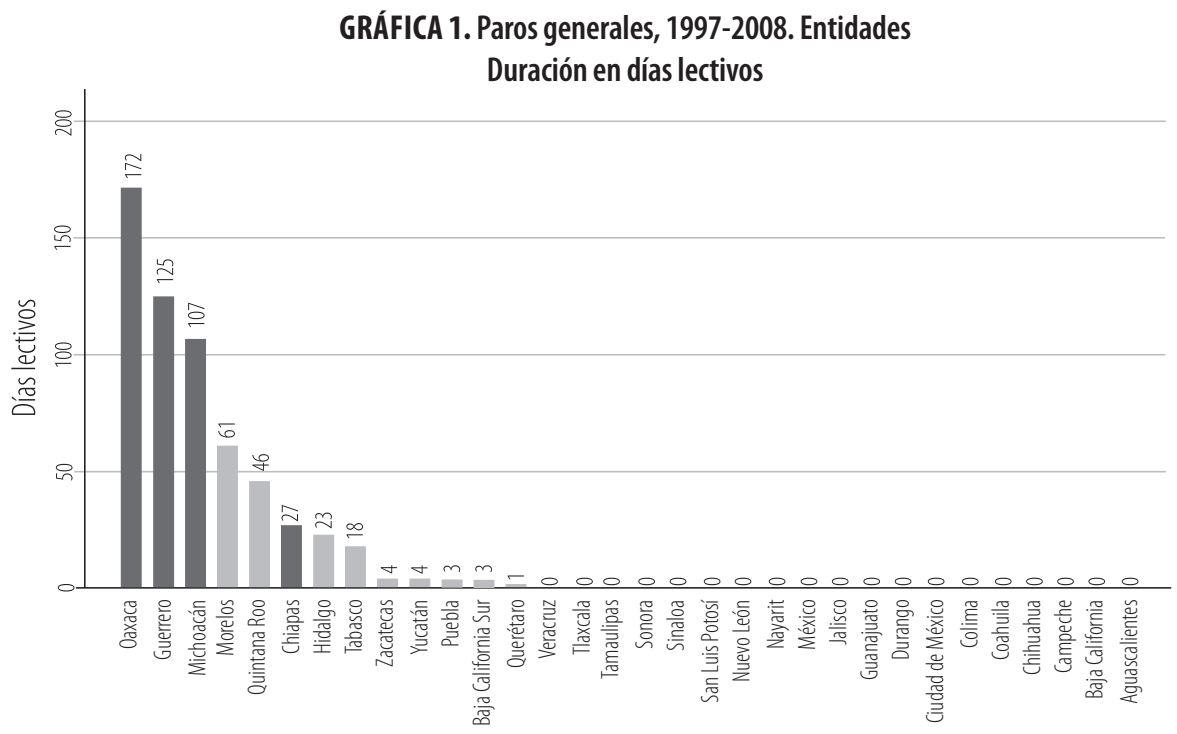

Fuente: Elaboración propia a partir de la revisión de prensa. 1996-2008.

En contraste, Oaxaca registra la presencia de una sección disidente que domina en la entidad y que además recurre a las movilizaciones como recurso de negociación frecuente, al menos hasta antes de que la reforma se empezara a implementar en el estado. ${ }^{20}$ Se trata entonces de casos extremos.

\section{RESULTADOS}

\section{La formación de docentes}

Como puede observarse en el cuadro 4, se midió el nivel máximo de estudios de docentes [nivesDnt] (en lo sucesivo los nombres de las variables se colocan entre corchetes). También se incluyó el número de cursos de actualización y/o de capacitación a los que habían asistido en el último ciclo [cursdnte]. ${ }^{21}$

${ }^{20}$ Se excluyeron casos como Guerrero, Michoacán, Morelos y otros porque son entidades en las que al interior de las secciones coexisten fracciones institucionales y disidentes, de modo que no se pueden catalogar propiamente de uno u otro modo.

${ }^{21}$ A este respecto cabe mencionar que se trataría de una situación atenuada por el programa de Carrera magisterial, que obliga a la asistencia a cursos a cambio de más salario. Sin embargo, se debe recordar que no todo el magisterio participa del programa, y que en enti- 
CUADRO 4. Reporte de medias y significancia estadística según ANOVAs

\begin{tabular}{|l|c|c|c|c|}
\hline \multicolumn{1}{|c|}{ Variable } & Privadas & S-22 & SS.II & Significancia \\
\hline Nivel máximo de estudios del docente (nivesDnt) & 3.47 & 3.26 & 3.36 & $*$ \\
\hline Número de cursos asistidos por el docente en el ciclo (cursdnte) & 3.51 & 2.87 & 3 & $*$ \\
\hline Índice de evaluación escolar del desempeño docente (levaldnte) & 4.41 & 4.05 & 4.08 & $*$ \\
\hline $\begin{array}{l}\text { El maestro dedica menos de la mitad del tiempo al aprendizaje } \\
\text { (mitadtpo) }\end{array}$ & 2.47 & 2.84 & 2.73 & $*$ \\
\hline El maestro sale del salón en horas de clase (saleaula) & 2.18 & 2.53 & 2.44 & $*$ \\
\hline Índice de compromiso docente (Icompdnte) & 4.25 & 4.15 & 4.22 & $*$ \\
\hline * $p<.05$ & & & & $*$ \\
\hline
\end{tabular}

Fuente: Elaboración propia

La comparación de medias mostró diferencias estadísticamente significativas (cuadro 3) en el nivel de estudios de los docentes de escuelas privadas (3.47) y de escuelas públicas, tanto oaxaqueñas (3.26) como de las SS.II. (3.36). También la prueba de Scheffe arrojó una diferencia estadísticamente significativa en el nivel máximo de estudios de los docentes de la S-22 y de las SS.II.

Por otro lado, se comparó el número de cursos promedio a los que los docentes asistieron en el último ciclo [cursdnte]. El resultado indicó la existencia de contrastes entre escuelas privadas (3.5 cursos), Oaxaca (2.9) y las SS.II. (3.0). ${ }^{22}$

\section{Rendición de cuentas}

Al comparar el índice de evaluaciones docentes a nivel de la escuela [Ievaldnte], el ANOVA indicó diferencias estadísticamente

dades como Oaxaca, hace varios años que el programa dejó de operar. Esto significa que en los hechos, la asistencia a cursos de capacitación estaría condicionada por factores distintos. Es en este espacio donde se puede sugerir un condicionamiento de tipo sindical, porque los maestros no solo buscan más salario, también buscan ascensos, movilidad, dobles plazas, préstamos, y una serie de bienes que muchas veces no se consiguen por el mérito en el aula, sino por la lealtad política.

${ }^{22}$ Aunque esto no resulta evidencia suficiente de un condicionamiento de tipo sindical, resulta un indicio de que, efectivamente, las escuelas públicas tienen algún condicionamiento que provoca menor asistencia de los docentes a cursos, y de manera más marcada sucede en Oaxaca que en las SS.II. Dada la perspectiva a propósito de los incentivos negativos para la formación docente, aquí se infiere la existencia de un condicionamiento que en algún grado sería de tipo sindical, sin asumir que lo sea de forma exclusiva. Factores como la orografía y las dificultades de traslado podrían abonar en la explicación del fenómeno. 
significativas entre las medias de las escuelas privadas (4.41), Oaxaca (4.05) y las SS.II. (4.08). Los datos no mostraron diferencias estadísticas en las escuelas públicas. Solo entre estas y las privadas.

\section{El tiempo lectivo}

Interesaba comparar el uso que los docentes hacen del tiempo lectivo en el aula. Para ello se analizaron dos preguntas de la base de alumnos, a saber, si el maestro usa menos de la mitad del tiempo a la enseñanza [mitadtpo] o si se ausenta del aula en horas lectivas [saleaula]. ${ }^{23}$

Se obtuvo que las escuelas privadas muestran una frecuencia media menor (2.47) en el uso de la mitad de tiempo para la enseñanza, esto en comparación con lo observado en Oaxaca (2.84) y en las SS.II. (2.73). La mayor frecuencia con la que los docentes emplean menos de la mitad del tiempo a la enseñanza en escuelas públicas resulta estadísticamente significativa.

Similar al factor previo, se observó un diferencia sensible en la frecuencia media con la que los maestros de escuelas públicas de Oaxaca (2.53) y de las entidades con SS.II. (2.45) salen del aula en horas lectivas; esto en comparación con los docentes de escuelas privadas (2.19). Nuevamente, se trata de diferencias estadísticamente significativas.

\section{Características de docentes}

El compromiso del docente resulta a la vez un término y un fenómeno difícil de observar y de medir. En el marco del presente estudio se generó un índice de compromiso [Icompdnte] a partir de siete variables. El ANOVA mostró diferencias estadísticamente significativas, con una ligera superioridad de las escuelas privadas (4.25) frente a Oaxaca (4.15) y a las SS.II. (4.22). También existe una diferencia estadísticamente significativa entre estas últimas y Oaxaca.

\footnotetext{
${ }^{23}$ La mayor pérdida de tiempo lectivo a causa de paros magisteriales en escuelas públicas resultaba obvia en comparación con las escuelas privadas donde no se observa el fenómeno. Sin embargo, dentro de las escuelas públicas de las secciones estudiadas resulta sobresaliente el caso de la S-22, cuyo contraste en términos de días perdidos a causa de los paros con las SS.II. es enorme (ver gráfica 1).
} 


\section{DISCUSIÓN DE RESULTADOS}

Los resultados obtenidos muestran diferencias en las medias del desempeño de maestros sindicalizados y no sindicalizados según el nivel máximo de estudios, el número de cursos al que asisten, la frecuencia con la que son evaluados en la escuela, el uso eficiente del tiempo lectivo, y el compromiso frente a los alumnos. Así también se observan diferencias en el desempeño de maestros afiliados a distintas secciones del SNTE, específicamente entre aquellos de secciones institucionales y los de secciones disidentes.

Estos resultados traen a colación la tercera pregunta que se planteó al inicio del texto (vid Supra): ¿existe algo que se pudiera denominar un efecto sindical sobre el desempeño de los docentes? O, ¿qué marca las diferencias entre las categorías de maestros estudiadas? ¿Importa el sindicato en el desempeño de sus afiliados en el aula?

Los resultados obtenidos no permiten ser concluyentes en estas cuestiones. Es necesario indagar mucho más en los determinantes del desempeño docente. En realidad, la cuestión analítica se puede plantear como la de entender los determinantes de un comportamiento en el aula y en la escuela. Se trata de una cuestión que hace eco de las preguntas y de la perspectiva planteada por el nuevo institucionalismo desde los ochenta, a saber, que el comportamiento de los individuos está afectado por los contextos, las normas, los valores y las organizaciones en las que interactúan (Manza, 2006). ${ }^{24} \mathrm{~A}$ este respecto, se tendría que discutir en futuros trabajos si el desempeño de los docentes en el aula es pensable bajo la perspectiva comportamentalista.

En general, los nuevos institucionalistas muestran cómo los valores, las normas, las ideas, las reglas, las rutinas y los roles que se derivan de los contextos guían o canalizan el comportamiento de los individuos (Nitta, 2007). Específicamente, los institucionalistas sociológicos argumentan que las estructuras y sistemas afectan a los individuos al mostrar cómo las expectativas, los roles y las rutinas se desarrollan en respuesta a un deseo de legitimidad y adecuación. También hacen hincapié en cómo las instituciones

\footnotetext{
${ }^{24}$ Una perspectiva planteada desde los sistemas de reglas e incentivos para los maestros se puede revisar en Taylor K., Koppich y Weeres, 1997.
} 
producen rutinas y roles que forman de manera generalizada el comportamiento individual (Nitta, 2007).

Por otro lado, los institucionalistas de la elección racional plantean que las instituciones dan forma a la acción porque ofrecen oportunidades de acción e imponen restricciones a la misma (Lecours, 2011). En esta perspectiva, la importancia teórica de las instituciones derivaría de su efecto mediador en los cálculos de los actores. Finalmente, y en respuesta a la elección racional, March y Olsen (1989) argumentan que el comportamiento es impulsado por otros elementos además de los cálculos de utilidad, por ejemplo, por los principios y valores internalizados, las características culturales, la identidad y el hábito. Los actores se comportan también para conformarse con las reglas o valores existentes.

Analizar el efecto de un sistema de reglas sobre el comportamiento de determinados actores resulta una perspectiva que no debería descartarse en los estudios sobre el desempeño docente. Sin embargo, en el marco del tema que aquí ocupa, resulta una tarea compleja y riesgosa dado el carácter multifactorial del desempeño. Bien se podría argumentar que este está condicionado por el contexto áulico o comunitario, o bien que existen condicionantes generadas por las autoridades educativas y no por el sindicato. ${ }^{25} \mathrm{La}$ validez de estas objeciones dota al ejercicio que aquí se reporta de un carácter preliminar o exploratorio que en ningún modo podría considerarse concluyente. No obstante, el comportamiento sistemático de ciertos rasgos de los docentes muestra la pertinencia del ejercicio ofrecido y de la agenda de investigación que abre.

Los resultados del ejercicio reportado mirados a la luz de la perspectiva planteada por el institucionalismo abren temas en los que estudios futuros deberían abundar: los docentes de escuelas públicas realizan su labor dentro de un marco normativo que condiciona su desempeño de forma distinta al de las escuelas públicas. La tarea es identificar el factor que introduce variaciones en los marcos normativos de los docentes o los procesos y procedimientos que configuran esos marcos. Una posibilidad que ha

\footnotetext{
${ }^{25}$ Además, no son pocas las críticas a la ineficiencia y a la corrupción de las autoridades educativas, cuya impericia puede referirse entre los factores que configuran el marco normativo en el que trabajan los maestros.
} 
de tratarse es justamente la del sindicalismo o lo que se ha enunciado como el efecto sindical.

En años recientes mucho se habló acerca de que el sistema de reglas de ingreso, permanencia y ascenso en el servicio docente había estado condicionado por la agenda laboral-sindical del SNTE. De esta manera, se planteaba que antes de la reforma, un maestro sindicalizado laboraba bajo reglas que privilegiaban la agenda laboral y política promovida por las dirigencias del SNTE, en detrimento de la agenda educativa (Muńoz A., 2008; OCE, 2008, 2009; Ornelas, 2008; Santibañez, 2008).

Una lectura crítica podría converger con los discursos antisindicales para plantear que, en los últimos años, en el sistema educativo mexicano prevaleció un conjunto de reglas que otorgaban estabilidad laboral sin méritos académicos, y que en el acceso a los beneficios/recursos del sistema educativo se premiaba más la lealtad al sindicato (en las SS.II.) o la participación y el activismo sindicales (en las secciones disidentes) que los méritos y el desempeño en las aulas. Según esta perspectiva, ello sería el resultado de un esquema de negociaciones cupulares entre las dirigencias del SNTE y las autoridades educativas, a través del cual estas últimas fueron perdiendo el control administrativo y académico del magisterio.

En la configuración de ese marco el SNTE no ha sido un actor único, pero sí un actor de peso, especialmente en los sexenios panistas (2000-2012), durante los cuales logró incidir marcadamente en los procesos de ingreso, ascenso y permanencia en el servicio docente y en la definición de reglas para la distribución de bienes en el sistema educativo.

En los últimos años el SNTE registró avances en la obtención de ventajas para su cúpula y sus agremiados, además de que durante los sexenios referidos aumentó su capacidad para influir o vetar la política educativa gracias al control de una estructura de representación monopólica, no competitiva, con presencia nacional y el liderazgo eficaz de Elba Esther Gordillo (Ornelas, 2008; Raphael, 2007).

Lejos de abandonar la función de representación, el SNTE asumió en los últimos ańos la búsqueda de una mayor cobertura del subsistema educativo y el incremento de los recursos públi- 
cos asignados al mismo, fuente inagotable de clientelas para las dirigencias.

También se debe destacar la versatilidad del SNTE y su dirigencia, así como el pragmatismo que le permitieron hacerse presente en el ámbito público a través de tres partidos políticos, uno de ellos resultado de su propia creación, además de controlar importantes ámbitos de la administración pública dentro y fuera del sector educativo, de donde se deriva una importante fuente de sus recursos políticos y materiales (Ornelas, 2012).

Todo lo anterior hizo del SNTE un tomador de decisiones relevante -e incluso predominante- en el sistema educativo. Bajo los gobiernos del PAN se constituyó inclusive como un actor con poder de veto que desplegaba diferentes estrategias para influir en las decisiones en materia educativa dependiendo si eran tomadas por el gobierno federal, por el congreso o por una administración estatal. Prueba de esto fueron las carteras que ocuparon en la SEP, en las gubernaturas y en los congresos (federal y locales) para desde ahí incidir en política educativa. Los pactos políticoelectorales fueron también parte de esto en cuanto le permitieron llegar a ámbitos de decisión de la política educativa. ${ }^{26}$

Otros trabajos (Bensusán y Tapia, 2014; Bensusán y Tapia, 2013) discutieron que la producción académica había abusado de las conjeturas en torno al SNTE y que se había perdido de vista el hecho de que se trataba de un organismo heterogéneo, y que dentro subsistían distintos modelos sindicales configurados al interior de sus secciones sindicales. De esta manera, resultaba factible suponer que a la heterogeneidad de modelos sindicales

\footnotetext{
${ }^{26}$ El SNTE adoptó como estrategia ocupar cargos públicos desde los cuales era posible incidir en la política educativa. Es ahora un lugar común afirmar que incidió en la política educativa desde el poder legislativo (principalmente el federal). Desde ahí se impulsó la ley que obliga al gobierno federal a destinar el $8 \%$ del PIB en educación (cifra que aún no se alcanza) y a hacer el preescolar de cobertura obligatoria (Santibañez, 2008). El sistema educativo resulta un campo política y económicamente rentable para quien lo controla, lo que no significa que los recursos que se destinan a este beneficien a los supuestos destinatarios. Es un sistema que distribuye bienes y recursos (educación, empleo, salario, seguridad social, etc.). Al respecto, Raphael (2007) detalla, con base en un informe de la propia SEP y las comparaciones de la OCDE, el lamentable estado de la infraestructura educativa y el bajo rendimiento escolar a pesar de los incrementos sustanciales en los recursos del sistema educativo y su aproximación a los porcentajes destinados en países desarrollados, aunque ello se explique en parte por los problemas creados con la federalización de la educación y la falta de coordinación entre las diversas instancias.
} 
correspondía una heterogeneidad de marcos normativos para el desempeño docentes. En otras palabras, las reglas del sistema educativo asumirían fisonomías diferenciadas en dos modelos de sindicalismo magisterial: el representado por las SS.II. y el de las secciones disidentes.

Los datos reportados indican que algunos de los componentes del desempeño docente están más condicionados por el modelo de la S-22 que por el de las SS.II. (Ver cuarta columna del cuadro 5). Esto último abona evidencia en el sentido de que se trata de modelos sindicales distintos, lo que de hecho refuerza la idea de que el SNTE no constituye un organismo homogéneo a lo largo del país; por el contrario, a su interior perviven secciones sindicales disímbolas en sus discursos, en sus estrategias, en sus efectos sobre el trabajo docente y en sus respuestas no solo ante la dinámica del sistema educativo, sino ante su reforma.

El cuadro 5 muestra una tendencia o patrón sistemático de resultados tal, que pone en duda la probabilidad de aleatoriedad. En otras palabras, la frecuencia de condicionamientos en docentes sindicalizados es tan frecuente, que es necesario plantear propuestas explicativas.

CUADRO 5. Relación de factores condicionados y no condicionados por el SNTE

\begin{tabular}{|l|l|l|l|}
\hline \multicolumn{1}{|c|}{ Variable } & \multicolumn{1}{|c|}{ Definición de variable } & \multicolumn{1}{c|}{ Status } & \multicolumn{1}{c|}{ Observación } \\
\hline nivesDnt & Nivel máximo de estudios del docente & Condicionado & Contraste en S-22 \\
\hline cursdnte & Número de cursos asistidos por el docente en el ciclo & Condicionado & Contraste en S-22 \\
\hline levaldnte & Índice de evaluación escolar del desempeño docente & Condicionado & \\
\hline mitadtpo & El maestro dedica menos de la mitad del tiempo al aprendizaje & Condicionado & \\
\hline saleaula & El maestro sale del salón en horas de clase & Condicionado & \\
\hline Icompdnte & Índice de compromiso docente & Condicionado & Contraste en S-22 \\
\hline
\end{tabular}

Fuente: Elaboración propia

Por otro lado, es justo advertir que la asociación entre sindicalismo y desempeño docente no es positiva ni negativa per se. Al tratarse de una asociación mediada por el sistema de reglas o el marco normativo que el sindicalismo co-configura, es posible que un modelo sindical distinto al que tomó forma en los años 
del corporativismo y del autoritarismo mexicanos (i. e. de los que resultó el SNTE) contribuyera en el diseño de reglas con efectos renovados en la labor docente.

Es oportuno advertir también que el ejercicio aquí presentado no se ha diseńado para mostrar la asociación de los rasgos de docentes con el aprendizaje. En otras palabras, el ejercicio no se puede tomar como evidencia de que los niveles de estudio de los docentes y/o su compromiso y uso del tiempo lectivo, etc., están asociados negativamente con los resultados de los alumnos. Esta sería una medición que aún debería realizarse. La evidencia aquí presentada no prueba que los rasgos de los docentes se convierten o no en aprendizaje.

Finalmente, la atención exclusiva a factores relativos al docente dejó de lado la asociación que podría presentar con la educación la opacidad en el uso o distribución de los recursos del sistema educativo, la corrupción, el diseño e implementación de determinadas políticas, y una serie de factores en los que se podría estudiar la imbricación del SNTE en el sistema educativo. Otras investigaciones tendrán que valorar el papel del Sindicato en esos campos y su posible efecto con los resultados educativos y con la labor de los maestros.

\section{CONCLUSIONES}

Se diseñó un ejercicio de medición del efecto sindical sobre algunos rasgos de docentes en los que teóricamente se esperaría observar una asociación o condicionamiento derivado del marco normativo que regula el trabajo docente. La medición se hizo comparando escuelas públicas y privadas, y secciones sindicales distintas.

De manera detallada, la evidencia sugiere que los docentes sindicalizados que laboran en primarias públicas generales, lo hacen con menores controles administrativos y menor número de evaluaciones del trabajo educativo por parte de sus directivos, además de que registran menores niveles de estudio y frecuentan menos cursos de formación continua que sus contrapartes no sindicalizadas de las escuelas privadas. Además, a decir de los alumnos, los maestros sindicalizados muestran un menor compromiso 
en su labor educativa, mientras que tienden a un uso deficiente del tiempo lectivo. Asimismo, los datos indican que algunos de estos rasgos son más marcados en los maestros afiliados a la S-22 de Oaxaca, que en los de las SS.II., lo que parece indicar que se trata de modelos sindicales que se asocian de forma distinta con el desempeño docente.

Es claro que en un contexto de polarización social como el que la reforma educativa ha generado, la evidencia ofrecida en el ejercicio reportado, aun sin ser conclusiva, puede prestarse a usos políticos. Para algunos sostendrá uno de los supuestos que guiaron la reforma educativa o al menos supondrá el reforzamiento de posturas antisindicales. Sin embargo, la única consecuencia que el ejercicio reportado asume como pertinente es acaso que las reglas con las que ha operado el sistema educativo en las últimas décadas y sus resultados, parecen indicar la necesidad de un cambio en las formas de representación y de promoción de los intereses del magisterio mexicano, es decir, la necesidad de ir hacia una renovación del sindicalismo docente.

\section{REFERENCIAS BIBLIOGRÁFICAS}

Addison, John T. y Hirsch, Barry T. "Union Effects on Productivity, Profits, and Growth: Has the Long Run Arrived?", en Journal of Labor Economics, 7(1), 1989, pp. 72-105, doi: 10.1086/298199.

Aidt, Toke y Tzannatos, Zafiris. Unions and Collective Bargaining. Economic Effects in a Global Environment, US, The International Bank for Reconstruction and Development/The World Bank, 168, 2002.

Allen, Steven G. "Trade Unions, Absenteeism, and Exit-Voice", en Industrial and Labor Relations Review, 37(3), 1984, pp. 331-345.

Andere, Eduardo. "Autoridades y sindicatos en plena encrucijada", en El Economista. Edición On Line, 2007. Disponible en http://eleconomista.com.mx/. Fecha de consulta, 08 de febrero de 2016.

Bensusán A., Graciela y Tapia G., Luis A. "Los problemas de la implementación de la 'reforma educativa'", en Gloria del 
Castillo y Giovanna Valenti N. (Eds.), Reforma educativa, ¿Qué estamos transformando? Debate informado, México, FLACSO Mexico, 2014, pp. 93-107.

Bensusán A., Graciela y Tapia G., Luis A. "El SNTE y el logro educativo. Una agenda de investigación en el contexto de la Reforma Constitucional”, en Revista Mexicana de Sociología, núm. 04/13, 2013.

Betcherman, Gordon. "The effect of unions on the innovative behaviour of firms in Canada", en Industrial Relations Journal, 22 (2), 1991, pp. 142-151, doi: 10.1111/j.14682338.1991.tb00633.x.

Bhattacherjee, Debashish. "Union-Type Effects on Bargaining Outcomes in Indian Manufacturing", en British Journal of Industrial Relations, 25 (2), 1987, pp. 247-266, doi: 10.1111/j.1467-8543.1987.tb00711.x.

Blanco B., Emilio E. Eficacia escolar en México. Factores escolares asociados a los aprendizajes en la educación primaria, (Tesis doctoral), México, FLACSO, 2007.

Boal, William M. y Pencavel, John. "The Effects of Labor Unions on Employment, Wages, and Days of Operation: Coal Mining in West Virginia”, en The Quarterly Journal of Economics, 109 (1), 1994, pp. 267-298, doi: 10.2307/2118435.

Braslavsky, Cecilia y Cosse, Gustavo. "Las actuales reformas educativas en América Latina: Cuatro actores, tres lógicas y ocho tensiones", en Revista Electrónica Iberoamericana sobre Calidad, Eficacia y Cambio en Educación (REICE), 4(2e), 2006, 26 pp. Fecha de consulta, 18 de julio de 2013.

Bronars, Stephen G., Deere, Donald R. y Tracy, Joseph S. “The effects of unions on firm behavior. An empirical analysis using firm-level data", en Industrial Relations, 33 (4), 1994, pp. 426-451, doi: 10.1111/j.1468-232X.1994.tb00350.x.

Brunello, Giorgio. "The Effect of Unions on Firm Performance in Japanese Manufacturing", en Industrial and Labor Relations Review, 45 (3), 1992, pp. 471-487.

Carini, Robert. "Teacher unions and student achievement", en Alex Molnar (Ed.), School reform proposals: the research evidence, US, NEPC, 2002, 24 pp. 
CCE. ¿Qué pasa con la calidad de la educación en México? Diagnóstico y propuestas desde la sociedad civil, México, Coalición Ciudadana por la Educación, 2010, 57.

Clark, Kim B. "Unionization and Firm Performance: The Impact on Profits, Growth, and Productivity", en The American Economic Review, 74(5), 1984, pp. 893-919.

Cook, Maria Lorena. Organizing dissent. Unions, the state, and the democratic teachers' movement in Mexico, University Park, Pa, Pennsylvania State University, 1996.

Coulson, Andrew J. "The effects of teachers unions on american education", en Cato Journal, 30(1), 2010, pp. 155-170.

Dan C., Alexander. Who's ruining our schools? The case against the NEA Teacher Union, US, Universidad de Michigan, 2008, 137.

Del Campo, Jesús Martín. "La CNTE: pasado y presente", en El Cotidiano (50), 1992, pp. 1-6.

Del Valle, Sonia, "Vigilan ciudadanos alianza educativa", Periódico Reforma, México, 22 de mayo de 2008.

Denny, Kevin. "Productivity and trade unions in British manufacturing industry 1973-85", en Applied Economics, 29 (10), 1997, pp. 1403-1409, doi: 10.1080/00036849700000030.

Eberts, Randall, Hollenbeck, Kevin y Stone, Joe. "Teacher performance incentives and student outcomes", en The Journal of human resources, 37(4), 2002, pp. 913-927.

Eberts, Randall W. y Stone, Joe A. "Teacher unions and the productivity of public school", en Industrial \& Labor Relations Review, 40(3), 1987, pp. 354-363.

Fernández A., Tabaré. Determinantes Sociales, Organizacionales e Institucionales de los Aprendizajes en la Educación Primaria de México: Un Análisis de Tres Niveles (2001), México, Instituto Nacional para la Evaluación de la Educación, 2003, 153 pp. Fuller, H. L., Mitchell, G. A. y Hartmann, M. E. "Collective Bargaining in Milwaukee Public Schools", en Tom Loveless (Ed.), Conflicting Missions? Teachers Unions and Educational Reform, US, Brookings Institution Press, 2000, pp. 110-149.

Gallardo, Marcela. "Obstáculos políticos en la implementación de las reformas educativas", en Formas y reformas de la educación, Chile, PREAL, Serie Políticas, vol. 5, 2000, 4 pp.). 
Gallardo, Marcela. "Sindicatos docentes y gobierno: consensuando las reformas educativas", en Formas y Reformas de la Educación, Chile, PREAL, Serie Mejores Prácticas, vol. 16, 2004, 4 pp.

Gallardo, Marcela. "La reforma latinoamericana entre dos clases políticas educativas", en Formas y reformas de la educación, Chile, PREAL, Serie Políticas, vol. 23, 2006, 4 pp.

Grimes, Paul W. y Register, Charles A. "Teachers' Unions and Student Achievement in High School Economics", en Journal of Economic Education, 21(3), (1990), pp. 297-306.

Hannaway, Jane y Rotherham, Andrew J. Collective Bargaining in Education and Pay for Performance (vol. 11), US, National Center on Performance Incentives, 2008, $31 \mathrm{pp}$.

Hess, Frederick M. y West, Martin R. A better bargain: Overhauling teacher collective bargaining for the 21st century, Harvard University, Program on Education Policy \& Governance, 2006, pp. 1-49.

Howard, Nelson, F. y Rosen, Michael. Are Teachers' Unions Hurting American Education? A State-by-State Analysis of the Impact of Collective Bargaining among Teachers on Student Performance, ERIC, Milwaukee, Institute for Wisconsin's Future, 1996, 25 pp.

Hoxby, Caroline Minter. "How Teachers' Unions Affect Education Production", en The Quarterly Journal of Economics, 111(3), 1996, pp. 671-718.

Ingersoll, Richard M., Hoxby, Caroline M. y Scrupski, Adam F., "Why Some Schools Have More Underqualified Teachers than Others", en Brookings Papers on Education Policy (7), 2004, pp. 45-88.

Johnson, Susan M. Teacher Unions in Schools, Philadelphia, Temple University Press, 1984, 253.

Kurth, Michael M. "Teachers' unions and excellence in education: An analysis of the decline in SAT scores", en Journal of Labor Research, VIII (4), 1987, pp. 351-394.

Lecours, André. "New institutionalism", en George Thomas Kurian (Ed.), The encyclopedia of political science (vol. 3), US, CQ Press, 2011, pp. 1107-1108. 
Luna A., Rocío y Arellanes M., Jesús. 30 años de la CNTE a través de sus congresos, Oaxaca, Comisión Estatal de Seguimiento y Concreción (Coesco)/Sección 22-SNTE, 2009.

Manza, Jeff. "New institutionalism", en Bryan S. Turner (Ed.), The Cambridge Dictionary of Sociology, US, Cambridge University Press, 2006, pp. 416.

March, James y Olsen, Johan P. Rediscovering Institutions. The Organizational Basis of Politics, New York, Free Press 1989.

Martínez, Nurit y Nieto, Francisco. "En educación, más calidad y controles”, en El Universal, México, 2013, p. 1.

Mexicanos, Primero y Fundación, Idea. Índice Compuesto de Eficacia de los Sistemas Escolares, México, Mexicanos Primero/Fundación IDEA, 2007, 327 pp.

Milkman, Martin. "Teachers' unions, productivity, and minority student achievement", en Journal of Labor Research, XVIII (1), 1997, pp. 134-150.

Moo, G. Gregory. Power grab: How the National Education Association is betraying our children, Regnery Publishing, 1999.

Muñoz A., Aldo. "Escenarios e identidades del SNTE. Entre el sistema educativo y el sistema político", en Revista Mexicana de Investigación Educativa, 13 (37), 2008, pp. 377-417.

Nitta, Keith A. "New institutionalism”, en Mark Bevir (Ed.), Encyclopedia of Governance, US, Sage Publications, 2007, p. 610.

OCDE. Teachers Matter. Attracting, Developing and Retaining Effective Teachers, France, OCDE, 2005, p. 237.

OCE. "La norma oficial de la educación: el SNTE contra la rectoría del Estado", en Este País, octubre (211), 2008, pp. 43-46.

OCE. "El SNTE y la política educativa", en Este País, septiembre (222), 2009, pp. 54-58.

Ornelas, Carlos. "El SNTE, Elba Esther Gordillo y el gobierno de Calderón", en Revista Mexicana de Investigación Educativa, 13 (37), México, COMIE, 2008, pp. 445-469.

Ornelas, Carlos. Educación, colonización y rebeldia: la herencia del pacto Calderón-Gordillo, México, Siglo XXI Editores, 2012.

Palamidessi, Mariano. Sindicatos docentes y gobiernos: conflictos y diálogos en torno a la reforma educativa en América Latina, Chile, PREAL, Programa de Promoción de la Reforma Educativa en América Latina y el Caribe, núm. 28, 2003, $35 \mathrm{pp}$. 
Raphael, Ricardo. Los socios de Elba Esther, México, Planeta, 2007.

Redacción. "Ven de gran importancia la reforma en educación", en Periódico El Universal, 03 de diciembre de 2013, p. 35.

Rentería, Mónica y Rubio, Emma. "Los clarouscuros de la democracia del Sindicato Nacional de Trabajadores de la Educación (SNTE)", en El Cotidiano (89), 1998, pp. 23-31.

Santibañez, Lucrecia. "Reforma educativa: el papel del SNTE", en Revista Mexicana de Investigación Educativa, 13 (abriljunio), México, COMIE, 2008, pp. 419-443.

SEP. Unidad de Planeación y Evaluación de Políticas Educativas, ENLACE Básica. Manual técnico, México, 2008.

Taylor K., Charles, Koppich, Julia E. y Weeres, Joseph G. United Mind Workers: Unions and Teaching in the Knowledge Society, US, Jossey-Bass, 1997.

Tiramonti, Guillermina. "Sindicalismo docente y reforma educativa en la América Latina de los 90", Chile, PREAL, Programa de Promoción de la Reforma Educativa en América Latina y el Caribe, núm. 19, 2001, 22 pp.

Tucker, Mark. Teachers, their unions and American education reform agenda, US, National Center On Education and the Economy, 2011, 13 pp.

Valenti, Giovanna, Blanco B., Emilio, y De los Heros, Martín. Factores asociados al logro educativo de matemáticas y español en la Prueba ENLACE, 2007: un análisis multinivel, México, FLACSO, 2007, 164 pp.

Valenti, Giovanna, Salazar E., Rodrigo, Florez, Nelson y Luna, Marisol. Factores asociados al logro educativo. Un enfoque centrado en el estudiante, México, FLACSO, 2009, 133 pp.

Vicente C., Joel. "El Movimiento Magisterial Oaxaqueńo. Una aproximación a sus orígenes, periodización, funcionamiento y grupos político-sindicales", en Joel Vicente C (Ed.), Educación, sindicalismo y gobernabilidad en Oaxaca, México, Editorial del Magisterio, 2006, pp. 33-86.

Villarino, Ángel. "Violan malos maestros derechos de los nińos", en Periódico Reforma, México, 11 de junio de 2014, p. 20.

Yescas M., Isidoro. "Movimiento magisterial y gobernabilidad en Oaxaca”, en El Cotidiano, 23(148), 2008, pp. 63-71. 
Yescas M., Isidoro y Zafra, Gloria. La insurgencia magisterial en Oaxaca, 1980, Oaxaca, México, Fondo Editorial Instituto Estatal de Educación Pública de Oaxaca, 2006, 139 pp.

Zigarelli, M. A. "The Linkages Between Teacher Unions and Student Achievement", en Journal of Collective Negotiations, 23, 1994, pp. 299-319. 


\title{
ANEXO 1
}

\section{Salida de CFA en Mplus para generar leva1dnte}

\author{
MODEL FIT INFORMATION \\ Number of Free Parameters \\ 18 \\ Loglikelihood

$\begin{array}{ll}\text { HO Value } & -118599.559 \\ \text { H1 Value } & -117873.848\end{array}$ \\ Information Criteria

$\begin{array}{lc}\text { Akaike (AIC) } & 237235.117 \\ \text { Bayesian (BIC) } & 237388.702 \\ \begin{array}{l}\text { Sample-Size Adjusted BIC } \\ \left(\mathrm{n}^{*}=(\mathrm{n}+2) / 24\right)\end{array} & 237331.498 \\ & \\ \begin{array}{l}\text {-Square Test of Model Fit } \\ \text { Value }\end{array} & 1451.421 \\ \begin{array}{l}\text { Degrees of Freedom } \\ \text { P-Value }\end{array} & 0.0000\end{array}$ \\ RMSEA (Root Mean Square Error Of Approximation) \\ Estimate $\quad 0.065$ \\ 90 Percent C.I. $\quad 0.0630 .068$ \\ Probability RMSEA $<=.05 \quad 0.000$ \\ $\mathrm{CFI} / \mathrm{TLI}$ \\ $\begin{array}{ll}\text { CFI } & 0.985 \\ \text { TLI } & 0.975\end{array}$ \\ Chi-Square Test of Model Fit for the Baseline Model \\ Value $\quad 96566.726$ \\ Degrees of Freedom 15 \\ P-Value $\quad 0.0000$ \\ SRMR (Standardized Root Mean Square Residual) \\ Value \\ 0.017
}

MODEL RESULTS

R-SQUARE

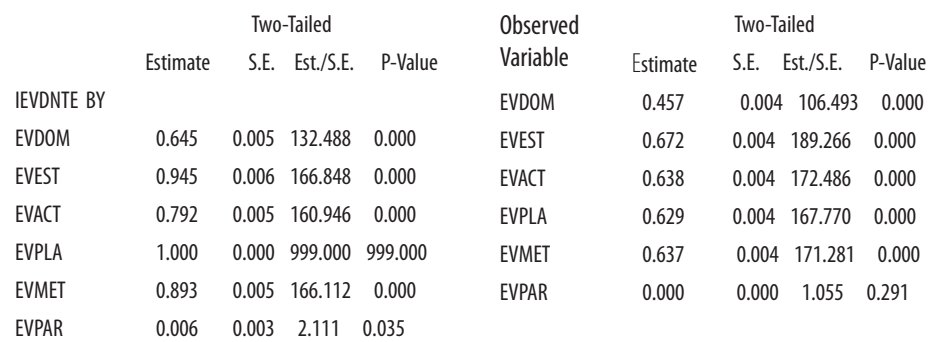




\section{ANEXO 2}

\section{Salida en CFA en Mplus para generar leva1dnt}

SUMMARY OF ANALYSIS

\author{
Number of groups \\ Number of observations
}

MODEL FIT INFORMATION

Number of Free Parameters

Chi-Square Test of Model Fit

Value

Degrees of Freedom

P-Value

RMSEA (Root Mean Square Error Of Approximation)

Estimate
90 Percent C.I.
Probability RMSEA $<=.05$

$\mathrm{CFI} / \mathrm{TLI}$

$$
\text { CFI }
$$

TLI

Chi-Square Test of Model Fit for the Baseline Model

Value

Degrees of Freedom

P-Value

WRMR (Weighted Root Mean Square Residual) Value

MODEL RESULTS

\begin{tabular}{|c|c|c|c|c|}
\hline & \multicolumn{2}{|c|}{ Two-Tailed } \\
\hline ICOMPD & Estimate & S.E. & Est./S.E. & P-Value \\
\hline P113| & 0.932 & 0.002 & 390.714 & 0.000 \\
\hline P114I & 0.968 & 0.002 & 407.023 & 0.000 \\
\hline P116I & 1.000 & 0.000 & 999.000 & 999.000 \\
\hline P117I & 0.958 & 0.002 & 389.037 & 0.000 \\
\hline P1201 & 0.840 & 0.003 & 319.833 & 0.000 \\
\hline P1211 & 0.749 & 0.003 & 272.191 & 0.000 \\
\hline P125I & 0.862 & 0.002 & 346.723 & 0.000 \\
\hline
\end{tabular}

1

205300

35

13102.508*

14

0.0000

0.067

0.0670 .068

0.000

0.982

0.973

721764.562

21

0.0000

11.336

$\begin{array}{llllll}\begin{array}{l}\text { R-SQUARE } \\ \text { Observed } \\ \text { Variable }\end{array} & \text { Estimate } & \text { S.E. } & \text { Est./S.E. } & \text { P-Value } & \text { Variance } \\ & & & & & \\ \text { P1131 } & 0.521 & 0.002 & 241.283 & 0.000 & 0.479 \\ \text { P1141 } & 0.562 & 0.002 & 261.472 & 0.000 & 0.438 \\ \text { P1161 } & 0.600 & 0.002 & 273.230 & 0.000 & 0.400 \\ \text { P1171 } & 0.551 & 0.002 & 237.559 & 0.000 & 0.449 \\ \text { P1201 } & 0.423 & 0.002 & 175.986 & 0.000 & 0.577 \\ \text { P1211 } & 0.337 & 0.002 & 144.613 & 0.000 & 0.663 \\ \text { P1251 } & 0.446 & 0.002 & 199.541 & 0.000 & 0.554\end{array}$

